\title{
Hearing Assessment-Reliability, Accuracy, and Efficiency of Automated Audiometry
}

De Wet Swanepoel, Ph.D., ${ }^{1,2}$ Shadrack Mngemane, B.Comm.Path., ${ }^{1}$ Silindile Molemong, B.Comm.Path., ${ }^{1}$ Hilda Mkwanazi, B.Comm.Path., ${ }^{1}$ and Sizwe Tutshini, B.Comm.Path. ${ }^{1}$

${ }^{1}$ Department of Communication Pathology, University of Pretoria, Pretoria, South Africa.

${ }^{2}$ Callier Center for Communication Disorders, School for Behavioral and Brain Sciences, University of Texas at Dallas, Dallas, Texas.

\section{Abstract}

Objective: This study investigated the reliability, accuracy, and time efficiency of automated hearing assessment using a computer-based telemedicine-compliant audiometer. Materials and Methods: Thirty normal-hearing subjects and eight hearing-impaired subjects were tested with pure-tone air conduction audiometry $(125-8,000 \mathrm{~Hz})$ in a manual and automated configuration in a counterbalanced manner. For the normal-hearing group each test was repeated to determine test-retest reliability and recording time, and preference for threshold-seeking method (manual vs. automated) was documented. Results: Test-retest thresholds were not significantly different for manual and automated testing. Manual audiometry test-retest correspondence was $5 \mathrm{~dB}$ or less in $88 \%$ of thresholds compared to $91 \%$ for automated audiometry. Thresholds for automated audiometry did not differ significantly from manual audiometry with $87 \%$ of thresholds in the normal-hearing group and $97 \%$ in the hearingimpaired group, corresponding within $5 d B$ or less of each other. The largest overall average absolute difference across frequencies was $3.6 \pm 3.9 \mathrm{~dB}$ for the normal-hearing group and $3.3 \pm 2.4$ for the hearing-impaired group. Both techniques were equally time efficient in the normal-hearing population, and 63\% of subjects preferred the automated threshold-seeking method. Conclusions: Automated audiometry provides reliable, accurate, and time-efficient hearing assessments for normal-hearing and hearing-impaired adults. Combined with an asynchronous telehealth model it holds significant potential for reaching underserved areas where hearing health professionals are unavailable.

Key words: e-health, telehealth, telemedicine, technology, audiometry, tele-audiology

\section{Introduction}

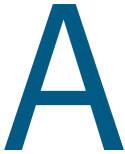

utomation of healthcare services is becoming increasingly important in light of the global shortage of specialized healthcare personnel. Automated or asynchronous healthcare services refer to a procedure or intervention conducted without requiring the necessary healthcare professional to be present. In situations where specialist healthcare personnel are limited or unavailable, this approach may ensure that services and healthcare resources are optimized. This is particularly relevant to hearing healthcare due to a high prevalence of hearing loss and a severe shortage of hearing healthcare professionals. ${ }^{1}$

Hearing loss is estimated to be the most prevalent disabling condition affecting approximately $10 \%$ of the global population to a mild or greater degree. ${ }^{2,3}$ Due to its high prevalence and significant consequences on quality of life, it is one of only four nonfatal conditions among the 20 leading causes of the global burden of disease. ${ }^{2}$ Unfortunately, the number of hearing healthcare professionals globally is inadequate to provide services to meet the need. ${ }^{4,5}$ For example, the majority of countries in Africa do not have audiology or otolaryngology services available. ${ }^{4}$ As a result, recent reports have highlighted the importance of developing tele-audiology services utilizing synchronous and asynchronous hearing assessment with integrated test automation to increase access to affordable and resource-efficient hearing healthcare. ${ }^{1,6-8}$ 
Typical diagnostic testing necessary to initiate appropriate intervention involves the characterization of hearing loss across frequency and intensity through pure-tone audiometry using airconducted stimuli. Including bone-conduction audiometry allows for differentiating conductive hearing loss contributions. Pure-tone audiometry is the primary procedure for assessing hearing in children and adults who are able to provide behavioral responses to sound, and it is typically performed by an audiologist or other hearing health professional. Since the early years of clinical audiometry, automation was considered because it is uniquely suited to audiometry due to the methodological nature of the procedures involved. ${ }^{7,9}$ Although limited studies in automated pure-tone audiometry are available, early studies reported good threshold correspondence with conventional manual audiometry. ${ }^{10-12}$ Clinically, however, it has not been included by audiologists as part of the diagnostic audiological test battery, and validation in hearing-impaired subjects is limited. ${ }^{7}$

Recent improvements in technology have made the application of automated audiometry significantly more feasible since these early reports. Devices can now be operated by laptop computers and data can be stored electronically while Internet connectivity facilitates centralized management and storage of data. Internet connectivity also allows for the test results to be forwarded for asynchronous review by remote specialists and can even facilitate synchronous hearing tests on patients in remote locations. ${ }^{1,6,8,13,14}$ The integration of this technology with features such as live monitoring of environmental noise and improved noise attenuation using combined insert earphones and circumaural earphones may ensure quality control during remote tests, whether synchronous or asynchronous. ${ }^{1,6}$ As a result, diagnostic audiological testing may even be conducted compliantly in remote areas where conventional infrastructure such as soundproof booths is often unavailable. ${ }^{1,6}$ A recent study demonstrated the accuracy of synchronous pure-tone audiometry using a telemedicine-compliant device with the remote clinician in Dallas, Texas, successfully testing a patient in South Africa. ${ }^{8}$

Automation of hearing tests in combination with asynchronous and synchronous telemedicine components has the potential to provide widespread access to services that are affordable and do not require specialist personnel onsite. ${ }^{1,8}$ The current study used a computer-based audiometer, recently used in the first intercontinental study of synchronous testing, ${ }^{8}$ to compare the reliability, accuracy, and efficiency of automated compared to manual audiometry.

\section{Materials and Methods}

The study was approved by the institutional ethics committee and all subjects were required to provide informed consent before par- ticipating. A sample of 30 normal-hearing subjects (age range, 18-31 years; average age, $22.5 \pm 3.4$ years; 18 women) and 8 hearingimpaired subjects (average age, $55 \pm 14$ years; 6 women) were recruited. Hearing thresholds for the normal-hearing group was $15 \mathrm{~dB}$ or less in 95\% of cases (397/420) compared to only 17\% of thresholds in the hearing-impaired population (19/112). Hearing losses in the hearing-impaired group were all sensorineural in nature and varied between mild and severe-to-profound degrees.

\section{EQUIPMENT}

An audiometer, recently demonstrated to be telemedicine compliant, ${ }^{8}$ with 3A insert earphones and additional circumaural earphone attenuation (KUDUwave 5000; GeoAxon), was used to measure pure-tone air conduction thresholds utilizing a software interface for testing and calibrated according to factory standards. The audiometer is software controlled and connected to a laptop via a USB port. The device is telemedicine compliant, allowing for synchronous testing, ${ }^{8}$ and is able to transmit results by e-mail to allow for asynchronous interpretation by remote clinicians. The audiometer's circumaural earphones are placed over insert earphones to increase the attenuation of environmental sound, while noise levels can be monitored by the device with an external microphone (on the circumaural earphone cup) and internal microphone (on inside of circumaural earphone cup) to ensure test compliance. This feature allows for quality control when synchronous or asynchronous testing is conducted especially in underserved areas where a soundproof booth may not be available. ${ }^{6}$

\section{MEASUREMENTS}

All pure-tone air conduction audiometry tests were conducted in a soundproof booth across octave-interval frequencies from 125 to $8,000 \mathrm{~Hz}$. All normal-hearing subjects (both ears) were tested with manual and automated threshold-seeking methods twice to determine test-retest reliability. The accuracy of automated audiometry was determined by comparing thresholds corresponding to those obtained with conventional manual audiometry. These comparisons were made between manual and automated thresholds obtained in conditions 1 and 2 for the normal-hearing group, which was the retest condition. The test order (manual vs. automated threshold seeking) was counterbalanced and all testing was conducted on the same day, but subjects were typically allowed a brief break between test procedures. Subjects were given basic instructions on what the test would entail and that they were required to press the response button each time they hear a signal in either ear.

A conventional $10 \mathrm{~dB}$ down and $5 \mathrm{~dB}$ up bracketing method (Modified Hughson-Westlake method) was used to determine 
pure-tone hearing thresholds. The test started in the left ear at $1,000 \mathrm{~Hz}$ (intensity, $30 \mathrm{~dB}$ hearing level [HL]) proceeding to higher octave frequencies, and after testing at $8,000 \mathrm{~Hz}$, lower octave frequencies were evaluated starting at $500-125 \mathrm{~Hz}$. In case of a no response at $30 \mathrm{~dB}$, the intensity was increased in $10 \mathrm{~dB}$ steps until a response was recorded and then the descending bracketing method was initiated again. Subjects indicated responses to the stimulus by using a hand-held response switch. The automated threshold-seeking paradigm used the same threshold-seeking method used in the manual test configuration. Software presented a tone for $1.25 \mathrm{~s}$ and participants had to respond within $1.5 \mathrm{~s}$ before the next tone could be presented. If a participant did not respond within the specific time, the system perceived it as a no-response and increased the intensity. Threshold was only accepted if there was a minimum of three responses. Software automatically determined if contralateral masking was necessary and if so, applied this as required in an adaptive manner.

Recording time and subject preference for threshold-seeking methods were also documented for manual and automated audi- ometry for normal-hearing subjects. The recording was initiated with the first stimulus presentation to the first ear tested and was terminated after the last subject response was recorded for the last ear tested. After the subjects with normal-hearing completed all four audiometric test procedures (two manual and two automated audiograms), they were asked to rate their preference for manual or automated threshold seeking. Three response options were possible: (1) preferred the automated test, (2) preferred the manual test, and (3) no preference.

\section{DATA ANALYSIS}

Descriptive measures illustrate the test-retest reliability of manual and automated audiometry and also indicate the difference in thresholds obtained between the two techniques. Two-sided paired $t$-tests were conducted across frequencies $(125-8,000 \mathrm{~Hz})$ to determine if there was a statistically significant difference between the results obtained within conditions for the normal-hearing group, that is, test-retest reliability, and the manual compared to automated test

\section{Table 1. Threshold Differences Between Test-Retest and Manual-Automated Audiometry Comparisons in Normal-Hearing Subjects ( $n=60$ Ears)

\begin{tabular}{l|l|l|l|l|l|l|l}
$125 \mathrm{~Hz}$ & $250 \mathrm{~Hz}$ & $500 \mathrm{~Hz}$ & $1,000 \mathrm{~Hz}$ & $2,000 \mathrm{~Hz}$ & $4,000 \mathrm{~Hz}$ & $8,000 \mathrm{~Hz}$ & OVERALL
\end{tabular}

Ave Abs difference $(\mathrm{dB})$

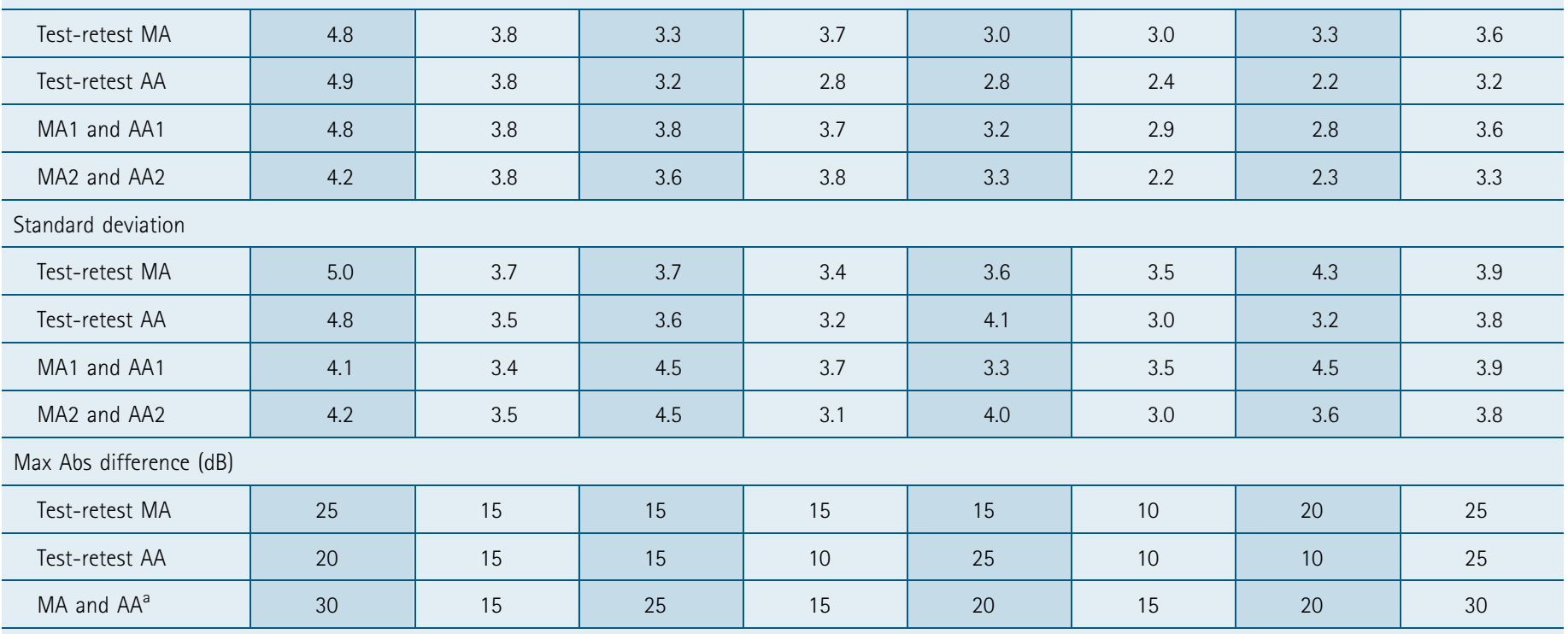

${ }^{a}$ Maximum absolute difference across all conditions (MA1 and $A A 1, M A 1$ and $A A 2, M A 2$ and $A A 1$, and MA2 and $A A 2$ ).

MA, manual audiometry; AA, automated audiometry; 1 and 2 refer to conditions 1 and 2; dB, decibel; Ave, average; Abs, absolute. 
Table 2. Overall Pearson Correlation Coefficients Between Thresholds Across Various Test Conditions

\begin{tabular}{|c|c|c|c|c|c|}
\hline & \multicolumn{4}{|c|}{$\begin{array}{l}\text { NORMAL-HEARING SUBJECTS } \\
\qquad(N=30)\end{array}$} & $\begin{array}{c}\text { HEARING- } \\
\text { IMPAIRED } \\
\text { SUBJECTS }(\boldsymbol{N}=\mathbf{8})\end{array}$ \\
\hline & $\begin{array}{l}\text { MA1 } \\
\text { AND } \\
\text { MA2 }\end{array}$ & $\begin{array}{l}\text { AA1 } \\
\text { AND } \\
\text { AA2 }\end{array}$ & $\begin{array}{l}\text { MA1 } \\
\text { AND } \\
\text { AA1 }\end{array}$ & $\begin{array}{l}\text { MA2 } \\
\text { AND } \\
\text { AA2 }\end{array}$ & $\begin{array}{l}\text { MA AND } \\
\text { AA }\end{array}$ \\
\hline $\begin{array}{l}\text { Correlation } \\
\text { coefficient (r) }\end{array}$ & 0.66 & 0.75 & 0.67 & 0.75 & 0.98 \\
\hline
\end{tabular}

conditions (criterion for significance at $p<0.05$ ). Pearson correlation coefficients $(r)$ between the various conditions were also determined.

\section{Results}

The test-retest threshold differences are presented in Table 1 and correlation coefficients in Table 2. The average absolute difference across all thresholds for test-retest conditions was similar, and the correlation was slightly better for automated (0.75) than for manual (0.66) audiometry in the normal-hearing group. As presented in Table 3, 88\% of all test-retest threshold comparisons $(368 / 420)$ for manual audiometry corresponded within $5 \mathrm{~dB}$ or less compared to $91 \%$ of automated audiometry thresholds (379/420). Only 3\% of

Table 3. Distribution of Threshold Correspondence for Test-Retest and Manual Compared to Automated Audiometry in Normal-Hearing Subjects ( $n=60$ Ears)

\begin{tabular}{|c|c|c|c|c|c|c|c|c|}
\hline & $125 \mathrm{HZ}$ & $250 \mathrm{HZ}$ & $500 \mathrm{HZ}$ & $1,000 \mathrm{HZ}$ & $2,000 \mathrm{HZ}$ & 4,000 HZ & $8,000 \mathrm{HZ}$ & OVERALL \% \\
\hline \multicolumn{9}{|c|}{$0 \mathrm{~dB}$ difference $(\%)$} \\
\hline Test-retest MA & 37 & 38 & 48 & 37 & 52 & 52 & 52 & 45 \\
\hline Test-retest AA & 37 & 35 & 47 & 52 & 55 & 57 & 65 & 50 \\
\hline MA1 and $A A 1$ & 30 & 38 & 47 & 43 & 45 & 52 & 63 & 46 \\
\hline MA2 and $A A 2$ & 42 & 38 & 47 & 33 & 50 & 62 & 63 & 48 \\
\hline \multicolumn{9}{|c|}{$5 \mathrm{~dB}$ difference $(\%)$} \\
\hline Test-retest MA & 42 & 50 & 40 & 57 & 38 & 37 & 35 & 43 \\
\hline Test-retest AA & 37 & 57 & 47 & 40 & 40 & 38 & 27 & 41 \\
\hline MA1 and $A A 1$ & 48 & 48 & 37 & 40 & 48 & 40 & 23 & 41 \\
\hline MA2 and $A A 2$ & 37 & 50 & 42 & 57 & 38 & 33 & 30 & 41 \\
\hline \multicolumn{9}{|c|}{$10 \mathrm{~dB}$ difference $(\%)$} \\
\hline Test-retest MA & 15 & 8 & 10 & 3 & 8 & 12 & 10 & 10 \\
\hline Test-retest AA & 20 & 5 & 3 & 8 & 3 & 5 & 8 & 8 \\
\hline MA1 and $A A 1$ & 17 & 13 & 12 & 17 & 5 & 7 & 8 & 11 \\
\hline MA2 and $A A 2$ & 18 & 10 & 8 & 10 & 10 & 5 & 3 & 9 \\
\hline \multicolumn{9}{|c|}{$\geq 15 \mathrm{~dB}$ difference $(\%)$} \\
\hline Test-retest MA & 7 & 3 & 2 & 3 & 2 & 0 & 4 & 3 \\
\hline Test-retest AA & 7 & 3 & 3 & 0 & 2 & 0 & 0 & 2 \\
\hline MA1 and $A A 1$ & 5 & 0 & 5 & 0 & 2 & 2 & 5 & 3 \\
\hline MA2 and $A A 2$ & 3 & 2 & 2 & 0 & 2 & 0 & 3 & 2 \\
\hline
\end{tabular}




\begin{tabular}{|c|c|c|c|c|c|c|c|c|}
\hline & $125 \mathrm{HZ}$ & $250 \mathrm{HZ}$ & $500 \mathrm{HZ}$ & $1,000 \mathrm{HZ}$ & $2,000 \mathrm{HZ}$ & $4,000 \mathrm{HZ}$ & $8,000 \mathrm{HZ}$ & OVERALL \\
\hline \multicolumn{9}{|c|}{ Ave Abs difference (dB) } \\
\hline \multicolumn{9}{|c|}{ Standard deviation } \\
\hline$M A$ and $A A$ & 3.2 & 2.4 & 2.6 & 2.6 & 2.6 & 3.1 & 3.1 & 2.8 \\
\hline $\mathrm{MA}$ and $\mathrm{AA}$ & 10 & 5 & 5 & 5 & 5 & 10 & 10 & 10 \\
\hline
\end{tabular}

thresholds differed by $>10 \mathrm{~dB}$ in the manual conditions $(4 / 12$ at $125 \mathrm{~Hz}$ ) and $2 \%$ of thresholds in the automated conditions $(3 / 9$ at $125 \mathrm{~Hz}$ ). There was no statistically significant difference between the test-retest threshold differences across frequencies $(125-8,000 \mathrm{~Hz})$ for manual and automated audiometry conditions ( $t$-test; $p>0.05$ ).

The average threshold differences between manual and automated audiometry (Table 1) across all frequencies were very small for conditions 1 and 2 in the normal-hearing group. The largest average absolute difference was at $125 \mathrm{~Hz}$ for condition 1 ( $4.8 \pm 4.1 \mathrm{~dB})$ and for condition $2(4.2 \pm 4.2 \mathrm{~dB})$, and the smallest differences was at higher frequencies $(4,000$ and $8,000 \mathrm{~Hz})$. Correlation coefficients for automated and manual audiometry in condition 1 were slightly lower (0.67) than for condition 2 (0.75). As presented in Table 3, 87\% of manual and automated thresholds in condition $1(362 / 420)$ corresponded within $5 \mathrm{~dB}$ or less of each other compared to $89 \%$ in con-

Table 5. Mean Bilateral Test Time for Manual

and Automated Audiometry in Normal-Hearing Group

( $n=30$ Recordings)

\begin{tabular}{l|r|r|r|r|r|c} 
& & & & & \multicolumn{3}{|c}{ DIFFERENCE } \\
\cline { 6 - 8 } & MA1 & AA1 & MA2 & AA2 & MA1-AA1 & MA2-AA2 \\
\hline $\begin{array}{l}\text { Ave time } \\
\text { (min) }\end{array}$ & 7.7 & 7.4 & 7.2 & 7.2 & 0.2 & -0.1 \\
\hline SD & 2.3 & 1.6 & 2.2 & 1.5 & 2.3 & 1.9 \\
\hline Min & 4.2 & 5.0 & 4.1 & 5.1 & -0.8 & -1.0 \\
\hline Max & 12.6 & 11.9 & 13.2 & 11.1 & 0.8 & 2.1 \\
\hline
\end{tabular}

SD, standard deviation. dition 2 (373/420). Only 2\% of threshold comparisons for manual versus automated audiometry in conditions 1 and 2 was $15 \mathrm{~dB}$ or higher.

The subgroup of subjects $(n=8)$ with hearing loss presented with average threshold differences between manual and automated audiometry of 1.4-3.3 dB across frequencies (Table 4). Perfect agreement $(0 \mathrm{~dB})$ between manual and automated threshold comparisons was evident in 56\% of threshold comparisons, while 97\% (105/108) corresponded within $5 \mathrm{~dB}$ or less. In only three instances (3\%) was a difference of $10 \mathrm{~dB}$ noted between manual and automated thresholds. In both manual and automated conditions thresholds were not obtained at maximum intensities at three frequencies. Two of these "no responses" corresponded between manual and automated audiometry; in the remaining two cases; a threshold was recorded at the maximum intensity of the audiometer using manual audiometry, while a "no response" was obtained using automated audiometry and vice-versa. As a result these "no responses" (4/112) could not be used for comparative purposes. The correlation coefficient for the subjects with hearing loss was 0.98 (Table 2).

Table 5 provides a distribution of the average time required for recording a bilateral audiogram across octave frequencies (125$8,000 \mathrm{~Hz}$ ) with manual and automated audiometry in the normalhearing group. The average duration was slightly less for automated audiometry in conditions 1 and 2 but was not statistically significant ( $t$-test, $p>0.05$ ). After completing the tests, 63\% of subjects indicated a preference for automated audiometry compared to $23 \%$ preferring manual audiometry and 14\% indicating no preference.

\section{Discussion}

Automated air conduction pure-tone audiometry demonstrated test-retest reliability equivalent to that of manual audiometry and 
similar to previously reported test-retest ranges for audiometry with various types of transducers. ${ }^{15}$ Although not statistically significant, test-retest correspondence for automated audiometry was slightly better than manual audiometry with a correspondingly higher correlation coefficient ( 0.75 vs. 0.66 ). A similar pattern was previously reported by Jerlvall et al. ${ }^{10}$ in their comparison of manual and automated pure-tone audiometry. ${ }^{10}$ The objective nature of the automated procedure means that threshold-seeking procedures and criteria for determining the threshold are consistently applied without bias. Although this should be the case in manual testing that follows a set threshold-seeking procedure and threshold criteria, there is still subjective decision making on the part of the clinician. As a result, automated audiometry may present with slightly better test-retest reliability based on group data and also avoid any possibility of variability between clinicians. ${ }^{10}$

Accuracy of automated audiometry was defined according to the correspondence to the gold standard of conventional manual audiometry. The threshold difference between automated and manual audiometry in the normal-hearing group was not statistically significant, and threshold correspondence (87\% and 89\% within $5 \mathrm{~dB}$ for conditions 1 and 2, respectively) was similar to that of test-retest correspondence for manual audiometry ( $88 \%$ within $5 \mathrm{~dB}$ ). The correlation coefficient between automated and manual audiometry in conditions 1 and 2 was similar and better than the test-retest correlation coefficient for manual audiometry, which underscores the reliability of automated audiometry. The subgroup of hearing-impaired subjects indicated an even closer correspondence between manual and automated thresholds than in the normal-hearing subjects, with 97\% corresponding within $5 \mathrm{~dB}$ or less. The correlation coefficient was correspondingly high (0.98) for this subsample, indicating a high degree of accuracy for automated audiometry in the hearing-impaired population. This may be attributed to the apparent awareness of internal noise levels with the double attenuation using insert and circumaural earphones in normal hearers, which is largely eliminated in the presence of reduced hearing sensitivity for hearing-impaired subjects.

The time required for manual and automated audiometry was similar, with an average of 7.2-7.7 min required to test both ears in the normal-hearing group. This similarity in test time was also reported in earlier studies that presented with similar average test durations. ${ }^{10,12}$ Interestingly, subjects preferred the automated audiometry threshold-seeking technique. This may be attributed to the predictable nature of the threshold presentations for this specific automated protocol. Changes to presentation of stimuli in a more arrhythmic configuration may in fact be more appropriate to avoid false-positive responses due to anticipation. ${ }^{11}$
Current findings demonstrate the reliability and accuracy of automated audiometry as a diagnostic tool in normal-hearing and hearing-impaired subjects in a sound booth. Automated audiometry provides a reliable, accurate, and time-efficient way of hearing assessment that offers the potential to be utilized in underserved areas where hearing health professionals are unavailable. Validation of test compliance outside a soundproof booth is still lacking, but the use of quality control measures, including live monitoring of background noise and attenuation in the ear canal, and active noise cancellation techniques holds significant promise. ${ }^{1,6}$ Integrated telehealth models, including automated audiometry that operates in an asynchronous configuration in conjunction with synchronous (real-time) components for consultation and advanced testing in complicated cases, could offer efficient and effective ways of addressing the global shortage in hearing healthcare.

\section{Acknowledgments}

We thank Dr. Dirk Koekemoer, Geoaxon Holdings, for providing the KUDUwave audiometer and for his invaluable technical support throughout the research study.

\section{Disclosure Statement}

No competing financial interests exist.

\section{REFERENCES}

1. Swanepoel D, Clark JL, Koekemoer D, Hall JW III, Krumm M, Ferrari DV, McPherson B, Olusanya B, Mars M, Russo I, Barajas J. Telehealth in audiologythe need and potential to reach underserved communities. Int J Audiol 2010;49:195-202.

2. World Health Organization. The global burden of disease: 2004 update. Geneva: World Health Organization, 2008.

3. World Health Organization. Primary ear and hearing care training manuals. Geneva: World Health Organization, 2006.

4. Fagan JJ, Jacobs M. Survey of ENT services in Africa: Need for comprehensive intervention. Global Health Action 2009;DOI: 103402/gha.v2i0.1932.

5. Goulios H, Patuzzi RB. Audiology education and practice from an international perspective. Int J Audiol 2008;47:647-664.

6. Swanepoel D, Olusanya BO, Mars M. Hearing healthcare delivery in sub-Saharan Africa-a role for tele-audiology. J Telemed Telecare 2010;16:53-56.

7. Margolis RH, Morgan DE. Automated pure-tone audiometry: An analysis of need, capacity and benefit. Am J Audiol 2008;17:109-113.

8. Swanepoel D, Koekemoer D, Clark JL. Intercontinental hearing assessment-a study in tele-audiology. J Telemed Telecare (In press).

9. Rudmose W. Automatic audiometry. In: Jerger J, ed. Modern developments in audiology. New York: Academic Press, 1963:30-75. 
10. Jerlvall $L$, Dryselius $H$, Arlinger $S$. Comparison of manual and computer-controlled audiometry using identical procedures. Scand Audiol 1983;12:209-213.

11. Picard M, llecki HJ, Baxter JD. Clinical use of BOBCAT: A first report on the reliability and validity of computerized pure-tone audiometry. Audiology 1988;27:234-246.

12. Harris DA. A comparison of computerized audiometry by ANSI, Békésy fixed frequency, and modified ISO procedures in an industrial hearing conservation program. J Aud Res 1980;20:143-167.

13. Krumm M, Ribera J, Klich R. Providing basic hearing tests using remote computing technology. J Telemed Telecare 2007;13:406-410.

14. Swanepoel D, Hall JW III. A systematic review of telehealth applications in audiology. Telemed J E Health 2010;16:181-200.

15. Stuart A, Stenstrom R, Tompkins C, Vandenhoff S. Test-retest variability in audiometric threshold with supraaural and insert earphones among children and adults. Audiology 1991;30:82-90.
Address correspondence to: De Wet Swanepoel, Ph.D.

Department of Communication Pathology University of Pretoria C/O Lynnwood \& Roper Pretoria 0002 South Africa

E-mail: dewet.swanepoel@up.ac.za

Received: October 8, 2009 Revised: November 30, 2009 Accepted: December 1, 2009 